\title{
Studies of LysSi3, a Gram-negative targeting endolysin with broad bactericidal activity
}

\author{
Tatyana Prudnikova ${ }^{1}$, Daria V Vasina ${ }^{2}$, Dmitry Loginov ${ }^{1}$ and Ivana Kuta Smatanova ${ }^{1}$ \\ ${ }^{1}$ N.F. Gamaleya National Research Centre for Epidemiology and Microbiology, Ministry of Health of the Russian Federation, 123098 \\ Moscow, Russia. \\ ${ }^{2}$ Laboratory of Structural Chemistry, Institute of Chemistry, Faculty of Science, University of South Bohemia in České Budějovice, \\ Czech Republic \\ talianensis@gmail.com
}

The antibiotic resistance incidence is alarmingly increasing in both human and veterinary medicine and is one of the major concerns of contemporary healthcare. Because most of the currently used antibiotics have natural analogs with similar native structures, AMRassociated genes are widely present in bacteria in the environment, and they can be easily distributed to clinically important strains through horizontal gene transfer. Such bacteria pose a threat primarily in hospitals, where infections of immunocompromised patients often end fatally. A small group of bacteria causing nosocomial infections are present in both the developed and developing world; it is called ESKAPE in abbreviation and is comprised of Enterococcus faecium, Staphylococcus aureus, Klebsiella pneumoniae, Acinetobacter baumannii, Pseudomonas aeruginosa, and other Enterobacteriaceae species. These pathogens represent the most significant threat among the so-called superbugs, which can rapidly acquire resistance to several classes of antibiotics and can cause a variety of nosocomial infections, mostly in the respiratory or urinary tract, but bloodstream, wound, and skin infections are also frequent. Overall, nosocomial infections result in increased mortality and morbidity rate in the affected patients.

For endolysins targeting Gram-negative bacteria the specific activity against a broad host range is a known phenomenon, however, the molecular mechanisms specifying their broad spectrum of action are obscure. There is still no global understanding, whether bacterial cell lysis with endolysins is determined by the action of the enzymatic activity of the proteins or by additional functional domains containing in their structure, or the action of endolysins against Gram-negative microorganisms is determined by the joint interaction of several distinct domains.

Here we propose to investigate in details the structural aspects of LysSi3 endolysin mechanism of action. The LysSi3 is a peptidoglycan hydrolyzing, lysozyme-like enzyme with predicted muramidase activity (GH24 family) and broad bactericidal activity against ESKAPE pathogens.

Keywords: Antibiotic resistance, endolysin, ESKAPE 